\title{
El proceso de búsqueda de empleo del alumnado finalista de la UVigo
}

\author{
Job search Process for the Uvigo finalist student body \\ María Reyes Fernández, María Dolores Dapía \\ Universidad de Vigo
}

\begin{abstract}
Resumen
En la actualidad las empresas seleccionan a su plantilla por su potencial más que por sus conocimientos. Por lo tanto, es imprescindible que el alumnado posea una visión realista del mercado laboral para incrementar su nivel de empleabilidad y aproveche su período educativo para nutrirse de los conocimientos y habilidades complementarios para afrontar con éxito una candidatura laboral. En este contexto, vamos a presentar una investigación que pretendió identificar el grado de implicación y el uso de los canales aplicados al proceso de búsqueda de empleo, y conocer las creencias sobre este tema del alumnado finalista de la Universidad de Vigo.

Palabras clave: búsqueda de empleo, transición escuelatrabajo, inserción laboral, universidad.
\end{abstract}

\begin{abstract}
Nowadays, companies select to his future staff according to his potential more that for his knowledge. Therefore, it is very important for the student body to have a realistic approach about the labor market to increase in this way his level of employability and to drawing up on complementary knowledge and skills should be used to facing successly an employment application. In this context we are presenting a research which claimed to identify the degree of involvement and the use of the channels in the process of job search, and to know the beliefs in this subject by finalist students of the University of Vigo.

Keywords: job search, school to work transition, personnel placement, university.
\end{abstract}

Actualmente en los procesos de selección se aplica un nuevo enfoque. Las empresas buscan en las candidaturas no sólo los conocimientos teóricoprácticos necesarios para desempeñar de forma adecuada un puesto de trabajo, sino también rasgos de inteligencia emocional que refuercen de manera integral las potencialidades futuras de un puesto de trabajo (Gómez Gras, 2006).

Las circunstancias que han roto el esquema tradicional de contratación, que valoraba fundamentalmente la formación académica tradicional (títulos universitarios) y la experiencia laboral en puestos similares al demandado, han sido la existencia de un entorno muy competitivo, tanto a nivel empresarial (multitud de empresas operando en el mismo sector) como a nivel profesional (cada vez son más y mejores las candidaturas a ocupar los puestos de trabajo vacantes), y la aplicación en el mercado laboral de la teoría neoliberal en la que el empleo para toda la vida en la misma empresa y/o en la misma ocupación se ha acabado.

Por lo tanto, es fundamental para el alumnado universitario conocer qué aptitudes y actitudes le van a ser requeridas por el mercado laboral para potenciarlas, adquiriendo así un perfil más atractivo para las empresas e incrementando su nivel de empleabilidad.

Pero, no sólo la adecuación de las candidaturas a los perfiles profesionales demandados por el mercado laboral influye en el éxito del proceso de inserción, la habilidad hacia la búsqueda de empleo también facilita que la incorporación al mercado de trabajo sea más satisfactoria. Las oportunidades de empleo de cualquier individuo son tanto o más una función de su capacidad de emplearse como del mercado laboral y de disponer de unas adecuadas estrategias en la búsqueda de ese empleo (Rosser, Suriá y Villegas, 2012).

Consecuentemente, el período educativo ya no puede entenderse sólo como una etapa en la que cada estudiante se forma en aspectos meramente académicos. Conviene aprovechar este tiempo de formación académica para nutrirse de los conocimientos y habilidades complementarios que después ayudarán a afrontar con mayor éxito una candidatura laboral. Son múltiples las alternativas que se presentan para enriquecer el curriculum: prácticas en empresas, becas y estancias en el extranjero, formación en búsqueda activa de empleo y competencias, etc.

La preparación para la búsqueda de un puesto de trabajo no debe iniciarse una vez finalizada la titulación. El momento idóneo es el período universitario, puesto que cada una de las alternativas expuestas anteriormente puede hacerse de modo simultáneo al estudio sin suponer demasiado esfuerzo para el alumnado, constituyendo un ahorro considerable de tiempo y ayudando a que la transición escuela-trabajo sea menos drástica al haber ya asumido muchas responsabilidades y tomado importantes decisiones.

La posibilidad del período educativo universitario como tiempo necesario de preparación hacia la mejora de la empleabilidad es lo que nos ha llevado a realizar la investigación aquí presentada, centrada en analizar el proceso de búsqueda activa de empleo de estudiantes finalistas, y no de egresados y egresadas, como es más frecuente encontrar en la literatura científica existente sobre inserción laboral (ANECA, 2009; Vidal García, 2003). 


\section{Objetivos}

- Conocer cuál es la situación de los y las estudiantes finalistas de la Universidad de Vigo en relación con su búsqueda de empleo.

- Establecer cuáles son los principales canales de búsqueda de empleo que están siendo utilizados o van a ser empleados en su proceso de búsqueda activa de empleo y las creencias acerca de su nivel de efectividad.

- Establecer las creencias que tiene el alumnado acerca de qué criterios emplean los agentes empleadores para analizar sus candidaturas.

- Detectar qué factores creen que facilitan su inserción laboral.

\section{Participantes}

Tras realizar un muestreo aleatorio, multietápico, estratificado por ámbito de conocimiento, titulación y campus, la muestra resultante estuvo constituida por un total de 365 estudiantes matriculados y matriculadas en el último curso de su carrera en el año 2011/2012 en la Universidad de Vigo, con representación de todas las titulaciones de primer y segundo ciclo impartidas en esta institución de educación superior.

Dichas titulaciones, a efectos de análisis posteriores, fueron agrupadas según los ámbitos de conocimiento que la ACSUG (Axencia de Calidade para o Sistema Universitario de Galicia) emplea en los estudios de inserción laboral que referidos a los titulados y tituladas por universidades gallegas viene publicando desde el 2004. Esta agrupación se presenta "en nueve grupos de estudio (cuatro grupos de Ciencias Sociales y Jurídicas y dos de Humanidades, además de las restantes áreas del Consejo de Coordinación Universitaria), formados en función de las competencias adquiridas por los egresados en sus estudios universitarios” (ACSUG, 2004, 47).

\section{Instrumento}

Para la recogida de datos se administró un cuestionario diseñado ad hoc, en base a cuestiones extraídas y/o adaptadas de encuestas empleadas en otros estudios (ACSUG, 2004; Espada, 2010; Figuera, 1994). Fue revisado por un grupo experto formado por dos orientadoras laborales universitarias y el jefe del departamento de orientación de un Centro Integrado de Formación Profesional.

El cuestionario se estructura en cuatro bloques: variables socio-demográficas, variables académicoprofesionales, información y formación laboral, y búsqueda activa de empleo (BAE). En este artículo se analizarán los resultados obtenidos en las 4 escalas presentes en este último apartado.

Las escalas referidas a la situación actual en relación con la búsqueda de empleo (escala $1, \alpha=0.76$ ) y al uso de los canales propios de esta búsqueda (escala 2, $\alpha=0.77$ ) presentan una serie de afirmaciones, con varias alternativas de respuesta, a las que cada estudiante debe responder eligiendo una de ellas: $0=$ No lo he hecho, $1=$ Tengo planes, pero espero a acabar la carrera y $2=$ Ya lo he hecho.
La escala que mide la percepción que tienen los y las estudiantes que están a punto de acabar sus estudios en la Uvigo, sobre los aspectos que tienen en cuenta los agentes empleadores a la hora de valorar sus candidaturas en futuros procesos de selección, está compuesta por 20 variables a considerar como $0=$ poco importante, $1=$ importante y $3=$ muy importante (escala $3, \alpha=0.82$ ).

Por último, la escala que mide los factores percibidos por el alumnado finalista como facilitadores de su inserción (escala 4, $\alpha=0.84$ ) tiene como alternativas de respuesta: $0=$ en desacuerdo y $1=$ de acuerdo.

\section{Resultados}

Los resultados obtenidos se describen agrupados en dos apartados; en el primero, se presentan los datos globales del conjunto de la muestra, y, en el segundo, se exponen las diferencias, cuando existen, atendiendo a tres variables: ámbito de conocimiento, campus y género.

\section{Consideraciones globales}

La situación actual en relación con la búsqueda de empleo de los y las estudiantes finalistas de la Universidad de Vigo -escala 1-, podemos describirla como de una baja implicación con una media de 0.91 $(\mathrm{S} x=0.39)$ sobre 2 .

Tal y como se observa en la Tabla 1, en 8 de las 13 situaciones presentadas, la respuesta mayoritaria fue que aún no habían iniciado la tarea descrita. Sólo en 3 de las restantes, sí lo habían hecho, y en 2, aunque no lo habían realizado, tenían planes al acabar la carrera. Por lo que se puede deducir que, a pesar de que están a punto de finalizar sus estudios, aún no se han planteado seriamente su proceso de búsqueda de empleo.

Tabla 1

Situación en relación a la búsqueda de empleo.

\begin{tabular}{lccc}
\hline Situaciones & $0(\%)$ & $1(\%)$ & $2(\%)$ \\
\hline Consultar docentes salidas & 36.2 & 34.5 & 29.3 \\
Consultar profesionales & 23.3 & 35.3 & 41.4 \\
Fuentes inf. empleo & 15.9 & 32.3 & 51.8 \\
Información dcho. laboral & 48.8 & 30.1 & 21.1 \\
Formación no exigida carrera & 26.6 & 38.9 & 34.5 \\
Prácticas/trabajos no exigidos & & & \\
Universidad, relac. estudios & 31.2 & 37.8 & 31 \\
Curriculum vitae & 15.9 & 19.5 & 64.7 \\
Carta presentación & 39.7 & 25.8 & 34.5 \\
Lista contactos personales & 54.5 & 26.3 & 19.2 \\
Participar procesos selección & 46 & 31 & 23 \\
Elaboración proyectos & 47.1 & 38.9 & 14 \\
Madurar creación empresa & 63.8 & 26.8 & 9.3 \\
Entrevista orientación laboral & 57.8 & 29.9 & 12.3 \\
\hline \multicolumn{2}{l}{ 0=No lo he hecho; 1=Tengo planes; 2=Ya lo he hecho }
\end{tabular}

En concreto, la acción mayoritariamente emprendida ha sido elaborar el curriculum vitae (64.7\%). Otras prácticas a destacar, con porcentajes superiores al $40 \%$, son: consultar con asiduidad fuentes de información sobre empleo y consultar con profesionales de mi carrera.

Por el contrario, las acciones pendientes de ejecutar hasta el momento de cubrir el cuestionario por 
aproximadamente la mitad de los y las estudiantes finalistas, son: madurar la idea de crear mi propia empresa, realizar una entrevista de orientación laboral, elaborar una lista de contactos personales, buscar información sobre derecho laboral y ejercitarme en la elaboración de proyectos o programas.

Son en las situaciones de realizar actividades de formación no exigidas en los estudios y realizar prácticas o trabajos (con o sin remuneración) no exigidos por la universidad, pero relacionados con el campo profesional, donde el porcentaje de respuesta mayor se recoge en la opción de respuesta «tengo planes, pero espero a acabar la carrera», aunque es muy similar a los encontrados en las otras dos opciones de respuesta.

En cuanto a la escala 2, referida al uso de los canales existentes en nuestro actual mercado de trabajo para la búsqueda de empleo, tampoco el resultado es muy alentador con una media de 0.61 sobre $2(S x=0.40)$, por lo que podemos establecer que el nivel de utilización de esos canales es muy bajo. Esta circunstancia se ve corroborada por el hecho de que en 9 de las 10 opciones presentadas, el porcentaje mayoritario de respuesta se agrupa en «no lo he hecho» (ver Tabla 2).

En ningún caso, la opción «ya lo he hecho» presenta un porcentaje mayor que las otras dos opciones de respuesta, variando su rango de elección entre el 0,5\% y el 28,5\%. Y sólo en un caso, responder anuncios de trabajo, la respuesta con un más alto porcentaje es «tengo planes, pero espero a acabar la carrera».

Tabla 2.

Canales para la búsqueda de empleo: uso y percepción de su efectividad.

\begin{tabular}{lcccc}
\hline $\begin{array}{l}\text { Canales } \\
\text { búsqueda de } \\
\text { empleo }\end{array}$ & 0 & 1 & 2 & $\begin{array}{c}\text { Efecti } \\
\text { vidad } \\
\text { (\%) }\end{array}$ \\
\cline { 2 - 5 } SEPE & 48.8 & 27.9 & 23.3 & 13.4 \\
Autocandidatura & 41.9 & 37.5 & 20.5 & 19.7 \\
Resp. anuncios & 34.5 & 37 & 28.5 & 25.5 \\
Poner anuncios & 65.8 & 26.8 & 7.4 & 6.8 \\
Conv. pública & 51.5 & 36.4 & 12.1 & 11 \\
ETTs & 48.2 & 27.9 & 23.8 & 18.4 \\
Bolsas Trabajo & 47.1 & 34.2 & 18.6 & 17.8 \\
Cont. personales & 47.1 & 26.3 & 26.6 & 24.4 \\
Autoempleo & 69.6 & 24.4 & 6 & 3.6 \\
Servicio UVigo & 98.6 & 0.8 & 0.5 & 0.5 \\
\hline
\end{tabular}

$0=$ No lo he hecho; $1=$ Tengo planes; $2=\mathrm{Y}$ a lo he hecho

Teniendo en cuenta que la falta de uso de los canales para la búsqueda de empleo es la característica que define a las distintas variables que forman esta escala, debemos señalar que los más usados para conocer el mercado laboral, dentro de sus bajos valores, son: responder anuncios de trabajo, usar la red de contactos personales, las Empresas de Trabajo Temporal, el Servicio Público de Empleo y la autocandidatura.

Destacan por su invisibilidad entre el alumnado el uso de los servicios de la Universidad de Vigo (98.6\%) y la opción del autoempleo como fórmula de inserción (69.6\%), con elevados porcentajes de respuesta en la alternativa «no lo he hecho», y que parece que no se han planteado hasta el momento.
Así mismo, a los y las estudiantes finalistas se les preguntaba acerca de cuáles creían que en un futuro próximo podrían ser los más efectivos en su búsqueda. En sus respuestas destacan por tener un porcentaje de respuesta superior al 20\%, responder anuncios de trabajo y la red de contactos personales, seguidos por autocandidatura, las ETTs, las bolsas de trabajo, el Servicio Público de Empleo y responder a una convocatoria pública, con porcentajes superiores al $10 \%$. Siguen presentando las respuestas más negativas, la posibilidad de asesorarse para crear una empresa (3.6\%) y el uso de los servicios universitarios (0.5\%) ver Tabla $2-$.

Como el mercado laboral es el ámbito en el que confluyen demandantes de trabajo (personas de dieciséis o más años que desean trabajar) y oferentes (ofertas de empleo de empresas y administraciones públicas), creímos conveniente averiguar qué era lo que creían los y las estudiantes finalistas que buscan las empresas en las candidaturas que son elegidas para ocupar un puesto de trabajo, por lo que construimos la escala 3 con 20 variables o criterios que suelen ser tenidos en cuenta cuando se lleva a cabo un proceso de selección (Tabla 3). En general, los y las estudiantes finalistas le otorgan un nivel de importancia mediobajo ( $\tilde{\mathrm{X}}=0,43$ sobre $2, \mathrm{Sx}=0.09$ ).

A pesar de ello, el alumnado finalista opina que los agentes empleadores buscan prioritariamente personas con sentido de la responsabilidad, capacidad de adaptación, conocimientos de idiomas y capacidad de aprendizaje, criterios que poseen un porcentaje superior al $80 \%$ en la opción de respuesta «muy importante». Por lo que la candidatura ideal sería la que poseyera estas competencias

Les siguen experiencia laboral previa, capacidad de trabajo en equipo y posesión de estudios universitarios con porcentajes superiores al 70\%.

Destaca, por tener el porcentaje más alto de respuesta en la opción «poco importante», el prestigio o el nombre del centro universitario (43\%).

Así mismo, tener un buen expediente académico (25.5\%), la aceptación de puestos inferiores (21.1\%) y facilitar la movilidad geográfica (14.5\%), para un porcentaje representativo de personas encuestadas no son importantes como criterios tenidos en cuenta por los agentes empleadores en los procesos de selección.

Por último, las personas participantes en esta investigación tuvieron que valorar una serie de factores que podían mejorar su futura inserción laboral (escala 4). La mayoría de personas encuestadas estaban de acuerdo en su importancia como agentes facilitadores de inserción ( $\widetilde{x}=0.72$ sobre $1, \mathrm{Sx}=0.18$ ) -ver Tabla 4 -. Sólo en tener una idea de negocio y en el prestigio de la universidad, el porcentaje superior se encontraba en la opción referida a su «desacuerdo». Así mismo, las respuestas que presentan las variables referidas al azar/suerte y a la financiación para crear una empresa, no presentan una tendencia clara.

Sobresalen por su altos valores superiores al $80 \%$ en la opción «de acuerdo», las categorías capacidad de adaptación, formación en idiomas, capacidad de aprendizaje, capacidad de trabajar en equipo, 
formación en informática, información sobre ofertas de empleo, realización de prácticas y capacidad de comunicación oral y escrita.

Tabla 3.

Criterios tenidos en cuenta por los agentes empleadores en sus procesos de selección.

\begin{tabular}{|c|c|c|}
\hline Criterios & Opciones respuesta & $\%$ \\
\hline Estudios & Poco importante & 3.9 \\
\hline \multirow{2}{*}{ universitarios } & Importante & 22.7 \\
\hline & Muy importante & 73.4 \\
\hline \multirow{3}{*}{$\begin{array}{l}\text { Expediente } \\
\text { académico }\end{array}$} & Poco importante & 25.5 \\
\hline & Importante & 33.5 \\
\hline & Muy importante & 41 \\
\hline Realización de & Poco importante & 12.2 \\
\hline \multirow[t]{2}{*}{ Máster/Posgrado } & Importante & 33 \\
\hline & Muy importante & 54.8 \\
\hline Otros cursos de & Poco importante & 10.6 \\
\hline \multirow{2}{*}{ especialización } & Importante & 31.5 \\
\hline & Muy importante & 57.9 \\
\hline Prácticas & Poco importante & 8.8 \\
\hline \multirow[t]{2}{*}{ realizadas } & Importante & 28 \\
\hline & Muy importante & 63.2 \\
\hline Experiencia & Poco importante & 5.6 \\
\hline \multirow[t]{2}{*}{ laboral previa } & Importante & 16.4 \\
\hline & Muy importante & 78 \\
\hline Conocimientos & Poco importante & 8.1 \\
\hline \multirow{2}{*}{ de informática } & Importante & 29.1 \\
\hline & Muy importante & 62.8 \\
\hline Conocimientos & Poco importante & 3.3 \\
\hline \multirow{2}{*}{ de idiomas } & Importante & 14.9 \\
\hline & Muy importante & 81.8 \\
\hline \multirow[t]{3}{*}{ Motivación } & Poco importante & 7.7 \\
\hline & Importante & 24.3 \\
\hline & Muy importante & 68 \\
\hline \multirow{3}{*}{$\begin{array}{l}\text { Movilidad } \\
\text { geográfica }\end{array}$} & Poco importante & 14.5 \\
\hline & Importante & 31.2 \\
\hline & Muy importante & 54.3 \\
\hline \multirow{3}{*}{$\begin{array}{l}\text { Aceptación } \\
\text { puestos inferiores }\end{array}$} & Poco importante & 21.1 \\
\hline & Importante & 36.9 \\
\hline & Muy importante & 42 \\
\hline \multirow{3}{*}{$\begin{array}{l}\text { Comunicación } \\
\text { oral y escrita }\end{array}$} & Poco importante & 7.5 \\
\hline & Importante & 24.1 \\
\hline & Muy importante & 68.4 \\
\hline \multirow{3}{*}{$\begin{array}{l}\text { Trabajo en } \\
\text { equipo }\end{array}$} & Poco importante & 3.3 \\
\hline & Importante & 20.5 \\
\hline & Muy importante & 76.2 \\
\hline \multirow[t]{3}{*}{ Aprendizaje } & Poco importante & 3.9 \\
\hline & Importante & 15.5 \\
\hline & Muy importante & 80.6 \\
\hline \multirow[t]{3}{*}{ Adaptación } & Poco importante & 4.2 \\
\hline & Importante & 13.5 \\
\hline & Muy importante & 82.3 \\
\hline \multirow[t]{3}{*}{ Responsabilidad } & Poco importante & 3.6 \\
\hline & Importante & 12.3 \\
\hline & Muy importante & 84.1 \\
\hline \multirow{3}{*}{$\begin{array}{l}\text { Iniciativa } \quad \mathrm{y} / \mathrm{o} \\
\text { creatividad }\end{array}$} & Poco importante & 8.9 \\
\hline & Importante & 27.1 \\
\hline & Muy importante & 64 \\
\hline Liderazgo / dotes & Poco importante & 14.1 \\
\hline de mando & Importante & 35.6 \\
\hline & Muy importante & 50.3 \\
\hline personal & Poco importante & 17.5 \\
\hline y/o profesional & Importante & 27.8 \\
\hline & Muy importante & 54.7 \\
\hline Prestigio & Poco importante & 43 \\
\hline universidad & Importante & 35.8 \\
\hline & Muy importante & 21.2 \\
\hline
\end{tabular}

Tabla 4

Factores facilitadores de inserción laboral.

\begin{tabular}{|c|c|c|}
\hline \multicolumn{2}{|c|}{ Criterios } & Porcentaje \\
\hline \multirow[t]{2}{*}{ Formación universidad } & Desacuerdo & 24.4 \\
\hline & Acuerdo & 75.6 \\
\hline \multirow[t]{2}{*}{ Expediente } & Desacuerdo & 35.4 \\
\hline & Acuerdo & 64.6 \\
\hline \multirow[t]{2}{*}{ Prácticas } & Desacuerdo & 19.1 \\
\hline & Acuerdo & 80.9 \\
\hline \multirow[t]{2}{*}{ Experiencia laboral } & Desacuerdo & 21.1 \\
\hline & Acuerdo & 78.9 \\
\hline \multirow{2}{*}{ Situación mercado } & Desacuerdo & 29.5 \\
\hline & Acuerdo & 70.5 \\
\hline \multirow{2}{*}{ El azar / la suerte } & Desacuerdo & 45.7 \\
\hline & Acuerdo & 54.3 \\
\hline \multirow[t]{2}{*}{ Personas conocidas } & Desacuerdo & 25.2 \\
\hline & Acuerdo & 74.8 \\
\hline \multirow[t]{2}{*}{ Empleo 2.0} & Desacuerdo & 31.6 \\
\hline & Acuerdo & 68.4 \\
\hline \multirow[t]{2}{*}{ Bolsas de empleo } & Desacuerdo & 27.5 \\
\hline & Acuerdo & 72.5 \\
\hline \multirow[t]{2}{*}{ Información ofertas } & Desacuerdo & 18.9 \\
\hline & Acuerdo & 81.1 \\
\hline \multirow[t]{2}{*}{ Movilidad } & Desacuerdo & 27.2 \\
\hline & Acuerdo & 72.8 \\
\hline \multirow[t]{2}{*}{ Irse al extranjero } & Desacuerdo & 29.7 \\
\hline & Acuerdo & 70.3 \\
\hline \multirow[t]{2}{*}{ Conoc. mercado } & Desacuerdo & 36.9 \\
\hline & Acuerdo & 63.1 \\
\hline \multirow[t]{2}{*}{ Técnicas BAE } & Desacuerdo & 29 \\
\hline & Acuerdo & 71 \\
\hline \multirow[t]{2}{*}{ Esfuerzo BAE } & Desacuerdo & 32 \\
\hline & Acuerdo & 68 \\
\hline \multirow[t]{2}{*}{ Orientación laboral } & Desacuerdo & 39.4 \\
\hline & Acuerdo & 60.6 \\
\hline Motivación & Desacuerdo & 21.1 \\
\hline & Acuerdo & 78.9 \\
\hline Idiomas & Desacuerdo & 13.3 \\
\hline & Acuerdo & 86.7 \\
\hline Informática & Desacuerdo & 18.4 \\
\hline & Acuerdo & 81.6 \\
\hline Máster & Desacuerdo & 27.2 \\
\hline & Acuerdo & 72.8 \\
\hline Otra formación compl. & Desacuerdo & 22.1 \\
\hline & Acuerdo & 77.9 \\
\hline Conocimiento salidas & Desacuerdo & 35.7 \\
\hline & Acuerdo & 64.3 \\
\hline Idea de negocio & Desacuerdo & 53.9 \\
\hline & Acuerdo & 46.1 \\
\hline Financiación empresa & Desacuerdo & 49 \\
\hline & Acuerdo & 51 \\
\hline Comun. oral y escrita & Desacuerdo & 19.3 \\
\hline & Acuerdo & 80.7 \\
\hline Trabajar en equipo & Desacuerdo & 14.7 \\
\hline & Acuerdo & 85.3 \\
\hline Aprendizaje & Desacuerdo & 13.6 \\
\hline & Acuerdo & 86.4 \\
\hline Adaptación & Desacuerdo & 12.3 \\
\hline & Acuerdo & 87.7 \\
\hline Iniciativa/ creatividad & Desacuerdo & 21,1 \\
\hline & Acuerdo & 78.9 \\
\hline Prestigio universidad & Desacuerdo & 55.8 \\
\hline & Acuerdo & 44.2 \\
\hline
\end{tabular}




\section{Diferencias atendiendo al ámbito de conocimiento, campus y sexo.}

Los resultados obtenidos tras aplicar un análisis de varianza (ANOVA) nos permitieron constatar lo siguiente (ver Tabla 5):

- El ámbito académico es fuente significativa de variación en las escalas 1 y 2, referidas al nivel de implicación en el inicio del proceso de transición a la vida activa y al empleo de los canales propios de la búsqueda activa de empleo.

- Para la variable campus se han encontrado diferencias en la escala referida al uso de los canales propios para la búsqueda de empleo (escala 2).

- No se han encontrado diferencias significativas en ninguna de las cuatro escalas en función del género.

Tabla 5

Diferencias en escalas atendiendo a ámbito, campus y género

\begin{tabular}{llcc}
\hline Factor & Escalas & F & Sig. \\
\hline Ámbito & Situación búsqueda & 2.72 & 0.006 \\
& Uso canales búsqueda & 4.61 & 0.000 \\
& Aspectos valorados selección & 1.97 & 0.051 \\
& Factores inserción & 0.71 & 0.680 \\
Campus & Situación búsqueda & 3.04 & 0.051 \\
& Uso canales búsqueda & 5.12 & 0.006 \\
& Aspectos valorados selección & 2.33 & 0.099 \\
\multirow{5}{*}{ Género } & Facilitadores inserción & 2.18 & 0.115 \\
& Situación búsqueda & 1.62 & 0.203 \\
& Uso canales búsqueda & 1.58 & 0.210 \\
& Aspectos valorados selección & 0.97 & 0.324 \\
& Facilitadores inserción & 2.71 & 0.101 \\
\hline
\end{tabular}

Realizados los post hoc que nos permitirán determinar las diferencias significativas encontradas prueba de Tukey-, podemos afirmar:

- La implicación en la búsqueda de empleo del alumnado finalista de las titulaciones pertenecientes a las Ciencias de la Salud es mayor frente a la de los y las estudiantes agrupados como Jurídico-social B (por ejemplo, Licenciatura en Derecho) y D (por ejemplo, Licenciatura en Comunicación Audiovisual).

- El uso de los canales propios de una búsqueda activa de empleo es mayor también entre los y las estudiantes de Ciencias de la Salud sobre el alumnado del ámbito Jurídico-social A (Derecho, Economía, ...). También los y las estudiantes del ámbito Jurídico-social C (por ejemplo, maestros/as) presentan un mayor dominio que los y las del ámbito Jurídico-social A.

- El uso de los canales propios para la búsqueda de empleo es mayor entre el alumnado finalista del campus de Ourense sobre el de Vigo.

\section{Conclusiones}

Las principales conclusiones que podemos extraer de los datos aquí presentados son:
- El alumnado finalista de la Universidad de Vigo entiende el período educativo como una etapa en la que principalmente hay que preocuparse por los aspectos meramente académicos, dejando el proceso de BAE como una tarea a iniciar al acabar la carrera.

- Consecuentemente, es palpable la falta generalizada de uso de los canales para la búsqueda activa de empleo.

- No obstante, destaca el alumnado finalista de las titulaciones pertenecientes al ámbito de las Ciencias de la Salud como el que más implicado está en su proceso de transición a la vida laboral.

- La cultura emprendedora no es una opción tenida en cuenta entre el alumnado finalista de la Uvigo.

- Existe una creencia generalizada acerca de la poca efectividad que poseen los servicios que la Universidad de Vigo posee para fomentar la empleabilidad de sus estudiantes y egresados/as, probable causa que justifica el escaso empleo que de ellos hacen.

- Los y las estudiantes son conscientes de la importancia de las competencias transversales y personales en el éxito del proceso de búsqueda de empleo, puesto que creen que son criterios que los agentes empleadores tienen en cuenta a la hora de seleccionar a sus plantillas, y por lo tanto su posesión les va a facilitar su futura inserción laboral.

\section{Referencias}

ACSUG (2004). Proxecto de inserción laboral dos titulados polo sistema universitario de Galicia 19962001. Santiago de Compostela: Autor.

ANECA (2009). Los procesos de inserción laboral de los titulados universitarios en España. Madrid: Autor. http://www.aneca.es/Documentos-ypublicaciones/Otros-documentos-de-

interes/Insercion-laboral

Espada, L. (dir.) (2010). Estudo sobre a transición do alumnado da Universidade de Vigo ao traballo. Vigo: Universidade de Vigo.

Figuera, P. (1994). La inserción socioprofesional del universitario/a. Tesis Doctoral. Universidad de Barcelona, http://www.tdx.cat/handle/10803/2359

España.

Gómez Gras, J.M. (2006). El trabajo de los universitarios. En C.J. Van-der Hofstadt y J.M. Gómez (coords). Competencias y habilidades profesionales para universitarios (pp. 7-37). Madrid: Ediciones Díaz de Santos.

Rosser, A.: Suriá, R. y Villegas, E. (2012). Análisis comparativo de estrategias de búsqueda de empleo de los estudiantes universitarios 2011/12 y 2012/13. En M.T. Tortosa, J.D. Álvarez y N. Pellín (Coords.). $X$ Jornadas de Redes de Investigación en Docencia Universitaria (pp. 37-47). Alicante: Universidad de Alicante.

Vidal García, J. (Coord.) (2003). Métodos de análisis de la inserción laboral de los universitarios. Madrid: Ministerio de Educación, Cultura y Deporte. 